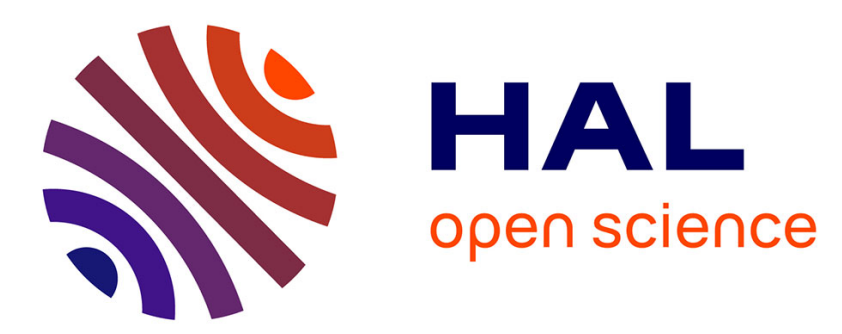

\title{
Inconsistency Management from the Standpoint of Possibilistic Logic
}

\author{
Didier Dubois, Henri Prade
}

\section{To cite this version:}

Didier Dubois, Henri Prade. Inconsistency Management from the Standpoint of Possibilistic Logic. International Journal of Uncertainty, Fuzziness and Knowledge-Based Systems, 2015, 23 (Suppl. 1), pp.15-30. 10.1142/S0218488515400024 . hal-01282037

\section{HAL Id: hal-01282037 https://hal.science/hal-01282037}

Submitted on 3 Mar 2016

HAL is a multi-disciplinary open access archive for the deposit and dissemination of scientific research documents, whether they are published or not. The documents may come from teaching and research institutions in France or abroad, or from public or private research centers.
L'archive ouverte pluridisciplinaire HAL, est destinée au dépôt et à la diffusion de documents scientifiques de niveau recherche, publiés ou non, émanant des établissements d'enseignement et de recherche français ou étrangers, des laboratoires publics ou privés. 


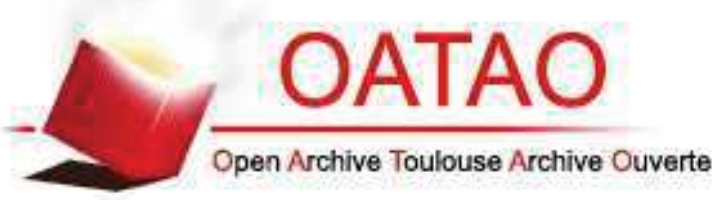

\section{Open Archive TOULOUSE Archive Ouverte (OATAO)}

OATAO is an open access repository that collects the work of Toulouse researchers and makes it freely available over the web where possible.

This is an author-deposited version published in : http://oatao.univ-toulouse.fr/ Eprints ID : 15084

To link to this article :

http://dx.doi.org/10.1142/S0218488515400024

To cite this version : Dubois, Didier and Prade, Henri Inconsistency

Management from the Standpoint of Possibilistic Logic. (2015)

International Journal of Uncertainty, Fuzziness and Knowledge-Based

Systems, Vol. 23 (Suppl. 1). pp. 15-30. ISSN 0218-4885

Any correspondance concerning this service should be sent to the repository administrator: staff-oatao@listes-diff.inp-toulouse.fr 


\title{
INCONSISTENCY MANAGEMENT FROM THE STANDPOINT OF POSSIBILISTIC LOGIC*
}

\author{
Didier Dubois and Henri Prade \\ IRIT-CNRS, Université Paul Sabatier, 31062 Toulouse Cedex 09, France
}

December 15, 2015

\begin{abstract}
Uncertainty and inconsistency pervade human knowledge. Possibilistic logic, where propositional logic formulas are associated with lower bounds of a necessity measure, handles uncertainty in the setting of possibility theory. Moreover, central in standard possibilistic logic is the notion of inconsistency level of a possibilistic logic base, closely related to the notion of consistency degree of two fuzzy sets introduced by L. A. Zadeh. Formulas whose weight is strictly above this inconsistency level constitute a sub-base free of any inconsistency. However, several extensions, allowing for a paraconsistent form of reasoning, or associating possibilistic logic formulas with information sources or subsets of agents, or extensions involving other possibility theory measures, provide other forms of inconsistency, while enlarging the representation capabilities of possibilistic logic. The paper offers a structured overview of the various forms of inconsistency that can be accommodated in possibilistic logic. This overview echoes the rich representation power of the possibility theory framework.
\end{abstract}

Keywords : inconsistency; fuzzy set; possibility theory; possibilistic logic.

\section{Introduction}

The intersection of two fuzzy sets may not be normalized. This state of fact may have several readings. In his founding paper, Lotfi Zadeh[35] already introduces the 
notion of degree of separation of two (convex) fuzzy sets $A$ and $B$, as the complement to 1 of the height ${ }^{1}$ of their min-based intersection. At that time, a fuzzy set was understood conjunctively as a collection of elements gathered into a class having an unsharp (gradual) boundary. Later, when Zadeh proposes to use fuzzy sets as a basis for representing possibility distributions[37], the understanding of a fuzzy set becomes disjunctive as an elastic restriction on the possible values of a single-valued variable. Then in this latter perspective, Zadeh[38] defines the consistency of two fuzzy sets as the height of their min-based intersection, without making an explicit use of it. However, in the same year, he defines the possibility of a fuzzy event[37], which is nothing but the degree of consistency between the fuzzy event and the possibility distribution representing what is known.

This idea of consistency is in full agreement with the classical logic notion of consistency between two propositions, which requires the existence of at least one common model for the two propositions. In such a case, Zadeh's consistency degree is equal to 1 , but his proposal extends the classical view by making it a matter of degree as soon as at least one of the two propositions is associated with a fuzzy set of models. Such a situation is encountered in possibilistic logic[20, 18, 24] where possibilistic logic formulas are semantically represented by means of a special type of possibility distributions. Moreover, the introduction of a consistency degree was also mentioned in relation with Zadeh's theory of approximate reasoning[39] based on the combination / projection of possibility distributions, which encompasses possibilistic logic inference[15, 16]. As it turned out, the degree of inconsistency, which is the complement to 1 of the degree of consistency, of a possibilistic logic knowledge base (made of a conjunction of possibilistic logic formulas) plays a great rôle in the possibilistic logic capability to handle inconsistency.

It can be observed that, in practice, a set of pieces of information is often inconsistent. Inconsistency often comes from the fact that the information is provided by different sources, but information provided by a person can be inconsistent as well. It may plainly take place between two opposite statements that are simultaneously held as certain. There are two basic ways to get around this problem: either one may restore consistency by isolating and mending parts of the information base judged to be responsible for the inconsistency; or one may alter the standard inference notion so as to make it more cautious, in order to preserve a consistent set of derived

\footnotetext{
${ }^{1}$ The notion of height of a fuzzy set (defined as the supremum of the membership degrees) was first introduced by Zadeh in his work on similarity relations[36] where he observes that for a $\max -\min$ transitive fuzzy relation, the height of the intersection of the fuzzy equivalence classes of two elements $x_{i}$ and $x_{j}$ is less or equal to their degree of similarity. This expresses that the more the equivalence classes overlap, the more similar their elements.
} 
conclusions.

However more subtle situations of inconsistency exist; for instance, if the representation setting is rich enough, an inconsistency may occur between the fact that something is known, while at the same time it is believed that it is not possible to know it. One may also have situations where apparently contradictory statements are in fact consistent once they are properly represented, as the statements "the museum is open in the morning", and the "the museum is open from 2 to 5 p.m". In any case, two contradictory statements cannot be simultaneously accepted as true. Then, rather than concluding, like in mathematics, that anything follows from a contradiction (the famous ex falso quodlibet sequitur), it is more useful to understand the origin and the nature of the inconsistency, and try to derive safe conclusions that overcome it.

Possibilistic logic[20, 18, 24] associates classical propositional formulas (and more generally first order logic formulas) with weights which may be lower bounds of different types of confidence evaluations making sense in possibility theory [37, 19]. The fact that possibility and necessity are graded provides additional power for handling inconsistency. This framework is expressive enough to represent various types of information, and may account for different situations of inconsistency. The paper surveys the existing works in possibilistic logic from an inconsistency-handling point of view. We first restate standard possibilistic logic where formulas are associated with lower bounds of necessity measures, before considering its extension to formulas having a graded paraconsistency level, or coming from different sources. We then additionally introduce formulas associated with lower bounds of weak or strong variant of possibility measures. This paper borrows some material from two conference papers $[22,26]$, and then merges it in an expanded overview.

\section{Possibility Theory and Possibilistic Logic}

In the following, formulas of a finite propositional language $\mathcal{L}$ will be denoted by Greek letters such as $\varphi$, or $\psi$. $\top$ and $\perp$ stand for tautology and contradiction respectively. For simplicity, we denote by $\Omega$ the set of interpretations of $\mathcal{L}$ that describe possible worlds; $\omega \models \varphi$ denotes the satisfaction of $\varphi$ by interpretation $\omega$, then called a model of $\varphi$. The set of models of $\varphi$ is denoted by $[\varphi]$. The negation of $\varphi$ is $\neg \varphi$. We also use conjunction and disjunction symbols $\wedge, \vee$. Finally classical syntactic inference is denoted by $\vdash_{C L}$. 


\subsection{Necessity and possibility measures}

A possibility distribution is a mapping $\pi$ from a set of possible worlds $\Omega$ to the interval $[0,1]$, which is viewed as a totally ordered bounded ordinal scale. Given a possible world $\omega \in \Omega, \pi(\omega)$ represents the degree of compatibility of $\omega$ with the available information (or beliefs) about the real world. $\pi(\omega)=0$ means that $\omega$ is impossible, and $\pi(\omega)=1$ means that nothing prevents $\omega$ from being the real world. When $\pi\left(\omega_{1}\right)>\pi\left(\omega_{2}\right), \omega_{1}$ is preferred to $\omega_{2}$ as a candidate for being the real state of the world. The less $\pi(\omega)$, the less plausible $\omega$, or the less likely it is the real world. A possibility distribution $\pi$ is said to be normalized if $\exists \omega \in \Omega$, such that $\pi(\omega)=1$, in other words, if at least one possible world is a fully plausible candidate for being the actual world. In that case, the knowledge represented by $\pi$ is considered to be consistent. Interpretations $\omega$ where $\pi(\omega)=1$ are considered to be normal (they are not at all surprising). A sub-normalized possibility distribution $\pi$ (such as height $(\pi)=\max _{\omega \in \Omega} \pi(\omega)<1$ ) is considered self-conflicting to some extent (since the existence of at least one fully possible interpretation is not acknowledged). The case where $\forall \omega, \pi(\omega)=0$ encodes a full contradiction. A consistent epistemic state is thus always encoded by a normalized possibility distribution.

Given a possibility distribution $\pi$, the possibility degree of proposition $\varphi$ is defined as:

$$
\Pi(\varphi)=\max \{\pi(\omega): \omega \models \varphi\} .
$$

It evaluates to what extent $\varphi$ is consistent with the possibility distribution $\pi$. Note that by definition if $\varphi \equiv \psi$ then $\Pi(\varphi)=\Pi(\psi)$, since $[\varphi]=[\psi]$. A necessity measure $N$ is always associated by duality with a possibility measure $\Pi$, namely

$$
N(\varphi)=1-\Pi(\neg \varphi)
$$

where $1-($.$) is the order-reversing map of the scale. The necessity measure N(\varphi)=$ $\min \{1-\pi(\omega): \omega \not \forall \varphi\}$ evaluates to what extent there does not exist a highly plausible interpretation that violates $\varphi$, in other words to what extent $\varphi$ can be deduced from the underlying possibility distribution $\pi$. Hence $N(\varphi)$ is a measure of the certainty of $\varphi$.

The duality between possibility and necessity extends the one in modal logic: it expresses that the impossibility of $\neg \varphi$ entails the certainty of $\varphi$. A necessity measure $N$ is a function from the set of logical formulas to the totally ordered bounded scale $[0,1]$, which is characterized by the axioms:

i) $N(\top)=1$,

ii) $N(\perp)=0$, 
iii) if $\varphi \equiv \psi$ then $N(\varphi)=N(\psi)$,

iv) $N(\varphi \wedge \psi)=\min (N(\varphi), N(\psi))$.

Axiom (iv) expresses that $\varphi \wedge \psi$ is as certain as the least certain of $\varphi$ and $\psi$. It follows from the axioms of necessity measures that having both $N(\varphi)>0$ and $N(\neg \varphi)>0$ forms a contradiction. In other words, one cannot be both somewhat certain of a proposition and of its negation. Moreover, one may have both $\Pi(\varphi)=1$ and $\Pi(\neg \varphi)=1$ without contradiction; it just acknowledges a state of (complete) ignorance about the truth value of $\varphi$.

\subsection{Possibilistic logic: Syntax}

We now recall the main features of possibilistic logic, before discussing some paraconsistent and multiple source extensions thereof in Sections 3 and 4. An atomic possibilistic logic formula[20] is a pair $(\varphi, a)$ made of a classical logic formula $\varphi$ and a positive real in $(0,1]$. The weight $a$ is interpreted as a lower bound for a necessity degree, i.e., the possibilistic logic formula $(\varphi, a)$ is semantically understood as the constraint $N(\varphi) \geq a$, where $N$ is a necessity measure. Note that formulas of the form $(\varphi, 0)$ do not contain any information (since for all $\varphi, N(\varphi) \geq 0$ always holds) and are not part of the language of possibilistic logic.

The min-decomposability of necessity measures allows us to work with weighted clauses without lack of generality, since $N\left(\bigwedge_{i=1, k} \varphi_{i}\right) \geq a \Leftrightarrow \forall i, N\left(\varphi_{i}\right) \geq a$, i.e., $\left(\bigwedge_{i=1, k} \varphi_{i}, a\right) \Leftrightarrow \bigwedge_{i=1, k}\left(\varphi_{i}, a\right)$.

The proof system of propositional possibilistic logic consists of axioms of propositional logic with weight 1 , and the following weighted modus ponens rule of inference:

$$
(\varphi, a),(\neg \varphi \vee \psi, b) \vdash(\psi, \min (a, b)) .
$$

where $\vdash$ denotes the syntactic inference in possibilistic logic. The following derived inference rules are valid in possibilistic logic:

- $(\neg \varphi \vee \psi, a),(\varphi \vee \rho, b) \vdash(\psi \vee \rho, \min (a, b))$

(resolution)

- $\forall b \leq a(\varphi, a) \vdash(\varphi, b)$

(weight weakening)

- if $\varphi \vdash_{C L} \psi$, then $(\varphi, a) \vdash(\psi, a)$

(logical weakening)

- $(\varphi, a),(\varphi, b) \vdash(\varphi, \max (a, b))$

(weight fusion) 
where $\vdash_{C L}$ denotes the classical logic entailment. Classical inference is retrieved when the weights are equal to 1 . Moreover $K \vdash(\varphi, a)$ if and only if $K_{a} \vdash_{C L} \varphi$, where $K_{a}$ is a classical logic base that is the $a$-level cut of the possibilistic logic base $K$, defined by $K_{a}=\{\varphi \mid(\varphi, b) \in K$ with $b \geq a\}$.

Finally, proving $(\varphi, a)$ from a possibilistic logic base $K$ also amounts to adding $(\neg \varphi, 1)$, put in clausal form, to $K$, and using the resolution rule repeatedly in order to show that $K \cup\{(\neg \varphi, 1)\} \vdash(\perp, a)$.

\subsection{Possibilistic logic: Semantics}

From a semantic viewpoint, a possibilistic logic base $K=\left\{\left(\varphi_{i}, a_{i}\right)\right\}_{i=1, \ldots, m}$ is associated with a possibility distribution $\pi_{K}$ representing the fuzzy set of models $\omega$ of $K$ :

$$
\pi_{K}(\omega)=\min _{i=1, \ldots, m} \max \left(\mu_{\left[\varphi_{i}\right]}(\omega), 1-a_{i}\right)
$$

where $\mu_{\left[\varphi_{i}\right]}$ is the characteristic function of the sets of models of $\varphi_{i}$. It can be shown that $\pi_{K}$ is the largest possibility distribution such that $N_{K}\left(\varphi_{i}\right) \geq a_{i}, \forall i=1, m$, i.e., the possibility distribution that allocates the greatest possible possibility degree to each interpretation in agreement with the constraints induced by $K$ (where $N_{K}$ is the necessity measure associated with $\pi_{K}$ ), namely

$$
\left.N_{K}(\varphi)=\min _{\omega \in[\neg \varphi]}\left(1-\pi_{K}(\omega)\right)\right) .
$$

Thus, a possibilistic logic base is associated with a fuzzy set of models. It represents the set of more or less plausible states of the world (according to the available information), when dealing with uncertainty. A possibility distribution which rankorders possible states is thus semantically equivalent to a possibilistic logic base. The semantic entailment is then defined by

$$
K \models(\varphi, a) \text { if and only if } N_{K}(\varphi) \geq a .
$$

It is also equivalent to $\forall \omega \pi_{K}(\omega) \leq \pi_{\{(\varphi, a)\}}(\omega)=\max \left(\mu_{[\varphi]}(\omega), 1-a\right)$. Indeed, $N_{K}(\varphi) \geq a$ is easily rewritten as $\pi_{K}(\omega) \leq 1-a$ if $\omega \models \neg \varphi$. It is worth noticing that $\pi_{K} \leq \pi_{\{(\varphi, a)\}}$ is nothing but the entailment principle in Zadeh's approach to approximate reasoning[39].

The syntactic inference machinery of possibilistic logic, using resolution and refutation, has been proved to be sound and complete with respect to the semantics[18]. Soundness and completeness are expressed by:

$$
K \vdash(\varphi, a) \Leftrightarrow K \models(\varphi, a)
$$




\subsection{Inconsistency level}

An important feature of possibilistic logic is its ability to deal with inconsistency. The level of inconsistency of a possibilistic logic base is defined as

$$
\operatorname{inc}(K)=\max \{a \mid K \vdash(\perp, a)\}
$$

(by convention $\max \emptyset=0$ ). We can explain this inconsistency level with the $a$-cuts: the inconsistency level of a base is the greatest value $a$ such that the corresponding $a$-cut is classically inconsistent. Clearly, any entailment $K \vdash(\varphi, a)$ with $a>\operatorname{inc}(K)$ can be rewritten as

$$
K^{c o n s, a} \vdash(\varphi, a),
$$

where

$$
K^{\text {cons }, a}=\left\{\left(\varphi_{i}, a_{i}\right) \in K^{\text {cons }} \text { with } a_{i} \geq a\right\}
$$

and

$$
K^{\text {cons }}=K \backslash\left\{\left(\varphi_{i}, a_{i}\right) \text { with } a_{i} \leq \operatorname{inc}(K)\right\} .
$$

$K^{\text {cons }}$ is the set of formulas whose weights are above the level of inconsistency. Thus they are not affected by the inconsistency, since more entrenched. Indeed, $\operatorname{inc}\left(K^{\text {cons }}\right)=0$, and more generally, inc $(K)=0$ if and only if the skeleton $K^{*}=$ $\left.\left\{\varphi_{i} \mid\left(\varphi_{i}, a_{i}\right) \in K\right)\right\}$ of $K$ is consistent in the usual sense. Moreover, it can be shown that

$$
\operatorname{inc}(K)=1-\max _{\omega} \pi_{K}(\omega)=1-\operatorname{height}\left(\pi_{K}\right) .
$$

It is important to observe that formulas $\varphi$ derived from $K$ with a level at most inc $(K)$ are drowned in the sense that $(\neg \varphi, i n c(K))$ can be derived as well. They cannot be inferred nor be used in a valid proof. It includes formulas $\varphi$ in $K$ whose declared certainty level is smaller or equal to $i n c(K)$, which cannot be sufficiently increased by deduction from $K$ (even if these formulas $\varphi$ do not belong to any minimal inconsistent subset of $\left.K^{*}\right)$. A way to partially escape the drowning effect is presented in the next section.

Lastly, let us also observe that if a possibilistic logic base $K$ contains two (fully) consistent sub-bases $C$ and $C^{\prime}$ (i.e., $C \subset K, C^{\prime} \subset K$, inc $\left.(C)=\operatorname{inc}\left(C^{\prime}\right)=0\right)$ ), such that $C \vdash(\varphi, a)$ and $C^{\prime} \vdash\left(\neg \varphi, a^{\prime}\right)$, then $\operatorname{inc}(K) \geq 1-\max \left(1-a, 1-a^{\prime}\right)=\min \left(a, a^{\prime}\right)$. Thus, $\operatorname{inc}(K)>0$ reveals the existence of consistent arguments in $K$ in favor of contradictory statements with certainty levels at least equal to $i n c(K)$. 


\section{$3 \quad$ Handling Inconsistency in Possibilistic Logic}

One may take advantage of the certainty weights for handling inconsistency in inferences, while avoiding the drowning effect (at least partially). We briefly survey two ways to cope with this problem.

\subsection{Degree of paraconsistency and safely supported-consequences}

An extension of the possibilistic inference has been proposed for handling inconsistent information and getting safely supported consequences[8] only. It requires the definition of a "paraconsistent completion" [15] of the considered possibilistic logic base $K$, as a first step. For each formula $\varphi$ such that $(\varphi, a)$ is in $K$, we extend the language and compute triples $(\varphi, b, c)$ where $b$ (resp. $c$ ) is the highest degree with which $\varphi$ (resp. $\neg \varphi$ ) is supported in $K$. More precisely, $\varphi$ is said to be supported in $K$ at least at degree $b$ if there is a consistent sub-base of $\left(K_{b}\right)^{*}$ that entails $\varphi$, where $K_{b}=\left\{\left(\varphi_{i}, a_{i}\right) \mid a_{i} \geq b\right\}$. Let $K^{o}$ denote the set of bi-weighted formulas thus obtained. $K^{o}$ is called the paraconsistent completion of $K$.

We call paraconsistency degree of a bi-weighted formula $(\varphi, b, c)$ the value $\min (b, c)$. In particular, the formulas of interest are such that $b \geq c$, i.e. the formula is at least as certain as it is paraconsistent. In particular, formulas such as $c=0$ are safe from any inconsistency in $K$. They are said to be free[8] in $K$.

Example 1 Take

$$
K=\{(\varphi, 0.8),(\neg \varphi \vee \psi, 0.6),(\neg \varphi, 0.5),(\neg \xi, 0.3),(\xi, 0.2),(\neg \xi \vee \psi, 0.1)\} .
$$

Note that inc $(K)=0.5$.

Then, $K^{\circ}$ is the set of bi-weighted formulas: $\{(\varphi, 0.8,0.5),(\neg \varphi, 0.5,0.8),(\neg \xi, 0.3,0.2),(\xi, 0.2,0.3),(\neg \varphi \vee \psi, 0.6,0),(\neg \xi \vee \psi, 0.6,0)\}$.

Consider for instance $(\neg \xi \vee \psi, 0.6,0)$. From $(\varphi, 0.8)$ and $(\neg \varphi \vee \psi, 0.6)$ we infer $(\psi, 0.6)$ (by modus ponens), which implies $(\neg \xi \vee \psi, 0.6,0)$ (by logical weakening); note that in this case this inference only uses formulas above the level of inconsistency (0.5). Besides, there is no way to derive $\neg \psi$ (nor $\varphi \wedge \neg \psi$ consequently) from any consistent subset of $K^{*}$; so $c=0$ for $\neg \xi \vee \psi$.

Remark 1 One may think of extending the paraconsistent completion $\mathcal{L}^{o}$ to the whole language $\mathcal{L}$ of $K$, in the spirit of the proposal made by Arieli[3] in the "flat" case (where the only certainty degrees are 1 and 0$): \forall \phi \in \mathcal{L}$ :

- $\phi \in \mathcal{L}_{T}$ if and only if there is a consistent subset of $K$ that entails $\phi$ and none that entails $\neg \phi$; and we can write $(\phi, 1,0) \in \mathcal{L}^{o}$. 
- $\phi \in \mathcal{L}_{F}$ if and only if there is a consistent subset of $K$ that entails $\neg \phi$ and none that entails $\phi$; and we can write $(\phi, 0,1) \in \mathcal{L}^{\circ}$.

- $\phi \in \mathcal{L}_{U}$ if and only if there is no consistent subset of $K$ that entails $\phi$ nor any that entails $\neg \phi$; and we can write $(\phi, 0,0) \in \mathcal{L}^{\circ}$.

- $\phi \in \mathcal{L}_{I}$ if and only if there is a consistent subset of $K$ that entails $\phi$ and another one that entails $\neg \phi$; and we can write $(\phi, 1,1) \in \mathcal{L}^{\circ}$.

In the above definition one can restrict to maximal consistent subbases of $K$. These four sets of formulas $\mathcal{L}_{T}, \mathcal{L}_{F}, \mathcal{L}_{U}, \mathcal{L}_{I}$ partition the language. It can be checked that $K^{o} \subset \mathcal{L}^{o}$. One can view the four annotations by pairs of Boolean values as akin to Belnap [7] epistemic truth-values, TRUE, FALSE, NONE and BOTH respectively. However, Belnap logic comes down to computing epistemic statuses of atomic propositions based on information from various sources, then obtaining the epistemic status of other formulas via truth-tables extending the usual ones to four values. See Dubois[12] and Dubois and Prade[25] for further discussions.

Clearly the formulas of the form $(\varphi, b, 0)$ in $K^{o}$ have an inconsistency level equal to 0 , and thus lead to safe conclusions. However, one may obtain a set of consistent conclusions from $K^{o}$, which is larger than the one that can be obtained from $K^{\text {cons }} \cup$ $K_{\text {free }}$ (where $K^{\text {cons }}$ denotes the set of formulas strictly above the inconsistency level, and $K_{\text {free }}$ the set of free formulas), as explained now.

Defining an inference relation from $K^{o}$ requires two evaluations:

- the undefeasibility degree of a consistent set $A$ of formulas:

$$
U D(A)=\min \left\{b \mid(\varphi, b, c) \in K^{o} \text { and } \varphi \in A\right\}
$$

- the unsafeness degree of a consistent set $A$ of formulas:

$$
U S(A)=\max \left\{c \mid(\varphi, b, c) \in K^{o} \text { and } \varphi \in A\right\}
$$

We say that $A$ is a reason for $\psi$ if $A$ is a minimal (for set inclusion) consistent subset of $K$ that implies $\psi$, i.e.,

- $A \subseteq K$

- $A^{*} \nvdash_{C L} \perp$

- $A^{*} \vdash_{C L} \psi$

- $\forall B \subset A, B^{*} \nvdash_{C L} \psi$ 
Then, let

$$
\begin{gathered}
U D(\phi)=\max \{U D(A): A \text { is a reason for } \phi\} \\
U S(\phi)=\min \{U S(A): A \text { is a reason for } \phi, U D(A)=U D(\phi)\} .
\end{gathered}
$$

The set of triples $(A, U D(A), U S(A))$ such that $A$ is a reason for $\psi$ is denoted by label $(\psi)$. Then, $(\psi, U D(\phi), U S(\phi))$ is said to be a DS-consequence of $K^{o}$ (or $K$ ), denoted by $K^{o} \vdash_{D S}(\psi, U D(\phi), U S(\phi))$, if and only if $U D(\phi)>U S(\phi)$ [8]. It can be shown that $\vdash_{D S}$ extends the entailment in possibilistic logic.

Example 2 (Example 1 continued): In the above example, label $(\psi)=\{(A, 0.6,0.5),(B, 0.2,0.3)\}$ with $A=\{(\varphi, 0.8,0.5),(\neg \varphi \vee \psi, 0.6,0)\}$ and $B=\{(\xi, 0.2,0.3),(\neg \xi \vee \psi, 0.6,0)\}$. Then, $K^{o} \vdash_{D S}(\psi, 0.6,0.5)$.

If we first minimize $U S(A)$ and then maximize $U D\left(A^{\prime}\right)$, the entailment would not extend the possibilistic entailment. Indeed in the above example, we would select $(B, 0.2,0.3)$ but $0.2>0.3$ does not hold, while $K \vdash(\psi, 0.6)$ since $0.6>\operatorname{inc}(K)=0.5$. Note that $\vdash_{D S}$ is more productive than the possibilistic entailment, as seen on the example, e.g., $K^{o} \vdash_{D S}(\neg \xi, 0.3,0.2)$, while $K \vdash(\neg \xi, 0.3)$ does not hold since $0.3<$ $\operatorname{inc}(K)=0.5$.

An entailment denoted by $\vdash_{S S}$, named safely supported-consequence relation, less demanding than $\vdash_{D S}$, is defined by $K^{o} \vdash_{S S} \psi$ if and only $\exists A \in \operatorname{label}(\psi)$ such that $U D(A)>U S(A)$. It can be shown that the set $\left\{\psi \mid K^{o} \vdash_{S S} \psi\right\}$ is classically consistent $[8]$.

This kind of inference can be also understood in terms of minimal inconsistent subsets[26]. Let $S$ be a minimal inconsistent subset in $K^{*}$, and let $\operatorname{inc}(S)=$ $\min \left\{a_{j} \mid\left(p_{j}, a_{j}\right) \in K, p_{j} \in S\right\}$ be the level of inconsistency of $S$. Then, observe that

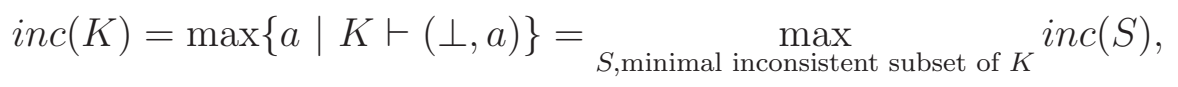

Moreover, it turns out that if $\left(p_{i}, \pi_{i}, \gamma_{i}\right) \in K^{o}$, we have

$$
\gamma_{i}=\max _{k: p_{i} \in C_{k}, C_{k} \text { minimal inconsistent subset of } K} \operatorname{inc}\left(C_{k}\right)
$$

with $\operatorname{inc}\left(C_{k}\right)=\min \left\{a_{j} \mid\left(p_{j}, a_{j}\right) \in K, p_{j} \in C_{k}\right\}$.

In fact, we have the following result: The safely supported entailment from $K$ coincides with the possibilistic entailment from the consistent possibilistic logic base 
$K_{\max }^{c o n s}$ obtained from $K$ by deleting, in all minimal inconsistent subsets $S$ of $K$, the formulas with a certainty level equal to $\operatorname{inc}(S)$. Namely

$$
K_{\max }^{\text {cons }}=K \backslash\left\{\left(p_{i}, a_{i}\right) \in S: S \text { minimal inconsistent subset of } K, a_{i}=\operatorname{inc}(S)\right\} .
$$

and we have

$$
K \vdash_{S S} \phi \Longleftrightarrow\left(K_{\max }^{c o n s}\right)^{*} \vdash_{C L} \phi
$$

\subsection{From quasi-classical logic to quasi-possibilistic logic}

Besnard and Hunter[10, 29] have defined a kind of paraconsistent logic, called quasiclassical logic. This logic has several nice features, in particular the connectives behave classically, and when the knowledge base is classically consistent, then quasiclassical logic gives almost the same conclusions as classical logic. ${ }^{2}$ Moreover, the inference in quasi-classical logic has a low computational complexity.

The basic ideas behind this logic is to use all rules of classical logic proof theory, but to forbid the use of resolution after the introduction of a disjunction (it allows us to get rid of the ex falso quodlibet sequitur). So the rules of quasi-classical logic are split into two classes: composition and decomposition rules, and the proofs cannot use decomposition rules once a composition rule has been used. Intuitively speaking, this means that we may have resolution-based proofs both for $\varphi$ and $\neg \varphi$. We also have as additional valid consequences the disjunctions build from the previous consequences (e.g. $\neg \varphi \vee \psi$ ). But it is forbidden to reuse such additional consequences for building further proofs[29].

It is clear that while possibilistic logic takes advantage of its weights for handling inconsistency, there are situations where possibilistic logic offers no useful answers, while quasi-classical logic does. This is when formulas involved in inconsistency have the same weight, especially the highest one, 1. For instance, consider the example $K=\{(\varphi, 1),(\neg \varphi \vee \psi, 1),(\neg \varphi, 1)\}$, where quasi-classical logic infers $\varphi, \neg \varphi, \psi$ from $K^{*}$, while everything is drowned in possibilistic logic, and nothing is obtained by the safely supported-consequence relation. This has led to propose a quasi-possibilistic logic[14] which has still to be further developed.

It would also have to be related to the simple generalized inference rule, applicable to formulas in $K^{o}$,

$$
(\neg \varphi \vee \psi, b, c)\left(\psi \vee \xi, b^{\prime}, c^{\prime}\right) \vdash\left(\psi \vee \xi, \min \left(b, b^{\prime}\right), \max \left(c, c^{\prime}\right)\right),
$$

proposed by Dubois et al.[15]. Note that in the above example, we would obtain $(\varphi, 1,1),(\neg \varphi, 1,1)$ and $(\psi, 1,1)$, as expected, by applying this rule. This rule can be

\footnotetext{
${ }^{2}$ In fact only tautologies or formulas containing tautologies cannot be recovered.
} 
viewed as the counterpart of the fact that in approximate reasoning the combination / projection principle provides, as consequences, fuzzy subsets whose height is the minimum of the heights of the fuzzy sets involved in the inference.

\section{Inconsistency Handling in Multiple Source In- formation}

In multiple source possibilistic logic[17], each formula is associated with a set (a fuzzy set more generally) which gathers the labels of sources according to which the formula is (more or less) certainly true. This leads to a simple extension of possibilistic logic, where propositions are associated not only with certainty levels, but also with the corresponding sources.

Consider, for instance, the following multi-source knowledge base where the information comes from sources $s_{1}, s_{2}, s_{3}$.

Example $3 K=\left\{\left(\neg \varphi \vee \psi,\left\{1 / s_{1}, 1 / s_{2}\right\}\right),\left(\neg \varphi \vee \xi,\left\{0.7 / s_{1}, 0.2 / s_{2}\right\}\right)\right.$,

$\left(\neg \psi \vee \xi,\left\{0.4 / s_{1}, 0.8 / s_{2}, 0.4 / s_{3}\right\}\right),\left(\neg \varphi \vee \neg \xi,\left\{0.3 / s_{3}\right\}\right)$,

$\left.\left(\varphi,\left\{0.5 / s_{1}, 0.8 / s_{2}, 0.5 / s_{3}\right\}\right),\left(\psi,\left\{0.8 / s_{1}, 0.9 / s_{2}\right\}\right),\left(\xi,\left\{0.6 / s_{2}\right\}\right)\right\}$.

Then by resolution and combination applied for each source, we can compute the multi-source certainty attached to $\xi$, for example. We obtain $N(\xi) \supseteq\left\{0.5 / s_{1}, 0.8 / s_{2}\right\}$ (where $\supseteq$ denotes fuzzy set inclusion, i.e. it means $N_{1}(\xi) \geq 0.5, N_{2}(\xi) \geq 0.8$ ), where $N_{i}$ is the ordinary necessity measure associated with source $i$, while $N$ is now a fuzzy set-valued extended necessity measure[17]. We can also prove $N(\neg \xi) \supseteq\left\{0.3 / s_{3}\right\}$, i.e. $N_{3}(\neg \xi) \geq 0.3$. Thus, the source $s_{3}$ is in conflict with $\left\{s_{1}, s_{2}\right\}$ with respect to $\xi$. But, by distinguishing between the sources, we avoid a global inconsistency problem. This idea can be further elaborated in connection with formal concept analysis [4] in order to associate subsets of sources to combination results obtainable from consistent subsets of pieces of information in an information merging process.

The idea of associating formulas with the sources that support them to some degree has been more systematically investigated in recent papers[21, 5], where formulas of the form $(\varphi, a / A)$ express that at least all agents in subset $A$ believe that $\varphi$ is true at least with certainty level $a$. Such formulas can be handled in a multi agent possibilistic logic where both the certainty levels $a$ and the subsets $A$ of agents are combined in the inference process. This enables us to distinguish between inconsistencies shared by some subsets of agents, and inconsistencies between beliefs held by disjoint subsets of agents. 


\section{$5 \quad$ Inconsistency with respect to Ignorance}

Standard possibilistic logic handles constraints of the form $N(\varphi) \geq a$. Constraints of the form $\Pi(\varphi) \geq a$ can be also considered, although they represent poorer pieces of information. Indeed $N(\varphi) \geq a \Leftrightarrow \Pi(\neg \varphi) \leq 1-a$ expresses partial certainty about $\varphi$, hence partial impossibility of $\neg \varphi$, while $\Pi(\varphi) \geq a$ only expresses that $\varphi$ true is somewhat possible. In particular, the state of (complete) ignorance about the truth value of $\varphi$ can be represented by $\Pi(\varphi)=1=\Pi(\neg \varphi)$, which states that both $\varphi$ and $\neg \varphi$ are fully possible.

Here appears another form of inconsistency between a statement of the form $N(\varphi) \geq a$ expressing that a proposition is somewhat certain, and a statement of the form $\Pi(\neg \varphi) \geq b$ (equivalently, $N(\varphi)<b$ ) expressing that the opposite proposition is somewhat possible, when the strict inequality $b>1-a$ holds between the degrees.

This situation is at work in the following cut rule[20], which mixes the two types of lower bound constraints on $\Pi$ and $N$, namely

$$
N(\neg \varphi \vee \psi) \geq a, \Pi(\varphi \vee \xi) \geq b \vdash \Pi(\psi \vee \xi) \geq a \& b
$$

with $a \& b=0$ if $a \leq 1-b$ and $a \& b=b$ if $a>1-b$. This type of inconsistency is of a higher level. It is a statement not dealing with the real world (e.g. claiming that one is sure that something is and is not), but a statement about epistemic states of external agents (agent 1 having reasons to believe that agent 2 is sure of something, and having reasons to believe that agent 2 is ignorant about this thing). This kind of knowledge can be expressed in generalized possibilistic logic[27], since one handles negations and disjunctions of standard possibilistic formulas, which allows contradictions of the form $N(\varphi) \geq a$ and $a \geq b>N(\varphi)$.

\section{Inconsistency in Bipolar Information}

The representation capabilities of possibilistic logic can be also enlarged in the bipolar possibilistic setting $[13,9]$. It allows the separate representation of both negative and positive information. Negative information reflects what is not (fully) impossible and remains potentially possible. It induces (prioritized) constraints on where the real world is (when expressing knowledge), which can be encoded by necessity-based possibilistic logic formulas. Positive information expressing what is actually possible, is encoded by another type of formula based on a set function called guaranteed (or actual) possibility measure (which is to be distinguished from "standard "possibility measures that rather express potential possibility (as a matter of consistency with the 
available information). This bipolar setting is of interest for representing knowledge and observations, and also for representing positive and negative preferences.

Positive information is represented by formulas denoted by $[\varphi, d]$, which expresses the constraint $\Delta(\varphi) \geq d$, where $\Delta$ denotes a measure of strong (actual) possibility[19] defined from a possibility distribution $\delta$ by $\Delta(\varphi)=\min _{\omega \models \varphi} \delta(\omega)$. This contrasts with a measure of (weak) possibility $\Pi$ which is max-decomposable, rather than min-decomposable (as $\Delta$ is) for disjunction.

Thus, the piece of positive information $[\varphi, d]$ expresses that any model of $\varphi$ is at least possible with degree $d$.

Let $D=\left\{\left[\varphi_{j}, d_{j}\right] \mid j=1, k\right\}$ be a positive possibilistic logic base. Its semantics is given by the possibility distribution

$$
\delta_{D}(\omega)=\max _{j=1, k} \delta_{\left[\varphi_{j}, d_{j}\right]}(\omega)
$$

with $\delta_{\left[\varphi_{j}, d_{j}\right]}(\omega)=0$ if $\omega \models \neg \varphi_{j}$, and $\delta_{\left[\varphi_{j}, d_{j}\right]}(\omega)=d_{j}$ if $\omega \models \varphi_{j}$. Thus, $\delta_{D}$ is obtained as the max-based disjunctive combination of the representation of each formula in $D$. This is in agreement with the idea that observations accumulate and are never in conflict with each other. Such a situation was already encountered in Mamdani and Assilian's fuzzy controllers[31, 23], where a weighted union of the contributions of each fuzzy rule that is fired, is performed.

A positive possibilistic knowledge base $D=\left\{\left[\varphi_{j}, d_{j}\right] \mid j=1, k\right\}$ is inconsistent with a negative possibilistic knowledge base $K=\left\{\left(\varphi_{i}, a_{i}\right) \mid i=1, m\right\}$ as soon as the following fuzzy set inclusion is violated:

$$
\forall \omega, \delta_{D}(\omega) \leq \pi_{K}(\omega)
$$

This violation occurs when something is observed while one is somewhat certain that the opposite should be true. Such an inconsistency should be handled by giving priority either to the positive or to the negative information[34].

\section{Concluding Remarks}

This overview has outlined the different forms of inconsistency that are expressible in possibility theory, when representing different types of information in a logical format. It is important to notice that the inconsistency, more precisely the contradictions here (see [11] on this point) may take place between different graded modalities. First, the same source cannot be certain at a positive degree of both $\varphi$ and $\neg \varphi$, i.e. contradictions between formulas is mirrored at the epistemic level in terms of necessity degrees. Two other forms of contradiction, either between asserted ignorance and certainty, or between what is reputed as being not possible and what 
is observed, involve two types of modalities. Contradictions can also take place in generalized possibilistic logic[27] at another level, since one handles negations and disjunctions of standard possibilistic formulas, as already seen above. Thus, the way inconsistency has to be managed depends not only from the application perspective (artificial intelligence inference systems vs. handling of dirty data in information systems $[32,30])$, but also of the nature of the inconsistency.

The paper has also pointed out the filiation existing between Zadeh's approximate reasoning theory and possibilistic logic. Although the two settings highly rely on possibility theory, it is interesting to notice that they have been developed in different directions and to try to understand why. Approximate reasoning theory mainly exploits the notion of possibility distribution and anticipates the representation of reasoning problems in terms of constraints (soft, in this case) which is at the basis of the constraint satisfaction problems that started to be investigated in artificial intelligence a decade later. The purpose of approximate reasoning following Zadeh was to represent and reason with pieces of fuzzy knowledge expressed in natural language, and encoded by possibility distributions on proper universes. Approximate reasoning is also closely related to fuzzy rule-based systems, but not so much to Mamdani's approach, since in this latter work, information is no longer viewed as constraints to be combined conjunctively, but rather as clues to be combined disjunctively[28]. But this important difference has remained almost unnoticed for a long time. Thus, approximate reasoning theory was based on possibility distributions and to some extent on possibility measures, while the other set functions of possibility theory (necessity, guaranteed possibility) were absent. ${ }^{3}$

On its side, possibilistic logic, while keeping a semantics in terms of possibility distributions (now defined on a set of interpretations, rather than on the domain of a linguistic variable) is much closer to classical logic; it is based on necessity measures and makes an extensive use of the degree of (in)consistency, whose expression formally appears in the first paper on fuzzy sets under the form of a separation degree between fuzzy sets, while generalized possibilistic logic accommodates all the modalities expressed by the set functions of possibility theory. Possibilistic logic is usually restricted to classical logic formulas, although there exist extensions to fuzzy propositions such as the one developed by Alsina and Godo[1, 2]. As in any logical

\footnotetext{
${ }^{3}$ This was partially counterbalanced by the introduction of the sophisticated notion of compatibility $[6,38,39]$ of a fuzzy set $G$ with respect to a fuzzy set $F$, defined as the fuzzy set of the possible values of the membership degree to $G$ of an element fuzzily restricted by $F$, which gives birth to the ideas of fuzzy truth values and fuzzy truth qualification. In fact, the compatibility both encompasses the consistency of $F$ and $G$ (or if we prefer the possibility of $G$ given $F$ ), and the necessity of $G$ given the fuzzy restriction expressed by $F[33]$.
} 
setting, inconsistency is a key notion in standard or generalized possibilistic logics, and may take various forms here due to the richness of the representation setting.

\section{References}

\section{References}

[1] T. Alsinet and L. Godo, "A complete calculus for possibilistic logic programming with fuzzy propositional variables", in Proc. 16th Conf. on Uncertainty in Artificial Intelligence (UAI'00), Stanford, (Morgan Kaufmann, San Francisco, 2000), pp. $1-10$.

[2] T. Alsinet, L. Godo, and S. Sandri "Two formalisms of extended possibilistic logic programming with context-dependent fuzzy unification: a comparative description", Elec. Notes in Theor. Computer Sci., 66 (5) (2002) 1-21.

[3] O. Arieli, "Conflict-tolerant semantics for argumentation frameworks", Proc. 13th Euro. Conf. Logics in Artificial Intelligence (JELIA12), eds. L. Fariñas del Cerro, A. Herzig and J. Mengin, Toulouse, Sept. 26-28, (Springer, LNCS 7519, 2012) pp. 28-40.

[4] Z. Assaghir, A. Napoli, M. Kaytoue, D. Dubois and H. Prade, "Numerical information fusion: Lattice of answers with supporting arguments", Proc. 23rd Inter. Conf. on Tools with Artificial Intelligence (ICTAI'11), Boca Raton, Nov.7-9, (IEEE, 2011) pp. 621-628.

[5] A. Belhadi, D. Dubois, F. Khellaf-Haned and H. Prade, "Multiple agent possibilistic logic", J. of Applied Non-Classical Logics, 23 (4) (2013) 299-320.

[6] R. E. Bellman and L. A. Zadeh, "Local and fuzzy logics", in Modern Uses of Multiple-Valued Logic, eds. J. M. Dunn and G. Epstein (D. Reidel, Dordrecht, 1977) pp. 103-165.

[7] N. D. Belnap, "A useful four-valued logic", in Modern Uses of Multiple-Valued Logic, eds. J. M. Dunn and G. Epstein (D. Reidel, Dordrecht, 1977) pp. 7-37.

[8] S. Benferhat, D. Dubois, and H. Prade, "An overview of inconsistency-tolerant inferences in prioritized knowledge bases", in Fuzzy Sets, Logic and Reasoning about Knowledge, vol.15 in Applied Logic Series, (Kluwer, 1999) pp. 395-417. 
[9] S. Benferhat, D. Dubois, S. Kaci and H. Prade, "Modeling positive and negative information in possibility theory", Int. J. of Intelligent Systems 23 (2008) 10941118.

[10] P. Besnard and A. Hunter, "Quasi-classical logic: Non-trivializable classical reasoning from inconsistent information", Proc. of the 3rd European Conference on Symbolic and Quantitative Approaches to Reasoning and Uncertainty (ECSQARU95), LNAI 946, (Springer Verlag, 1995) pp. 44-51.

[11] W. A. Carnielli, M. E. Coniglio and J. Marcos, "Logics of formal inconsistency", Handbook of Philosophical Logic, vol. 14, 2nd edition, eds. D. Gabbay and F. Guenthner, (Springer, 2007) pp. 1-93.

[12] D. Dubois, "On ignorance and contradiction considered as truth-values", Logic J. of the IGPL 16 (2): (2008)195-216.

[13] D. Dubois, P. Hajek and H. Prade, "Knowledge-driven versus data-driven logics", J. of Logic, Language, and Information 9 (2000) 65-89.

[14] D. Dubois, S. Konieczny and H. Prade, "Quasi-possibilistic logic and its measures of information and conflict", Fundamenta Informaticae 57 (2-4) (2003) $101-125$.

[15] D. Dubois, J. Lang and H. Prade, "Handling uncertainty, context, vague predicates, and partial inconsistency in possibilistic logic", in Fuzzy Logic and Fuzzy Control (Proc. of the IJCAI'91 Workshop on Fuzzy Logic and Fuzzy Control, Aug. 1991), eds. D. Driankov, P. W. Eklund and A. L. Ralescu, LNCS 833, (Springer-Verlag, 1994) pp. 45-55.

[16] D. Dubois, J. Lang and H. Prade, "Fuzzy sets in approximate reasoning Part 2: Logical approaches", Fuzzy Sets and Systems 40 (1991) 203-244.

[17] D. Dubois, J. Lang and H. Prade, "Dealing with multi-source information in possibilistic logic", Proc. of the 10th Europ. Conf. on Artificial Intelligence (ECAI'92) Vienna, Aug. 3-7, (Wiley, New York, 1992) pp. 38-42.

[18] D. Dubois, J. Lang and H. Prade, "Possibilistic logic", Handbook of Logic in Artificial Intelligence and Logic Programming, Vol. 3, eds. D. M. Gabbay, C. J. Hogger, J. A. Robinson, D. Nute, (Oxford University Press, 1994) pp. 439-513. 
[19] D. Dubois and H. Prade, "Possibility theory: qualitative and quantitative aspects", in Quantified Representation of Uncertainty and Imprecision, eds. D. Gabbay, P. Smets, eds., Handbook of Defeasible Reasoning and Uncertainty Management Systems, (Kluwer Acad. Publ., 1998) vol.1, pp. 169-226.

[20] D. Dubois and H. Prade, "Possibilistic logic: a retrospective and prospective view", Fuzzy Sets and Systems 144 (2004) 3-23.

[21] D. Dubois and H. Prade, "Toward multiple-agent extensions of possibilistic logic", Proc. IEEE Inter. Conf. on Fuzzy Systems (FUZZ-IEEE 2007) London, July 23-26, 2007, pp. 187-192.

[22] D. Dubois and H. Prade, "Handling various forms of inconsistency in possibilistic logic", Proc. 1st Int. Workshop on Data, Logic and Inconsistency (DALI11), in Database and Expert Systems Applications (DEXA) International Workshops (F. Morvan, A Min Tjoa, R. Wagner, eds.), Toulouse, Aug. 29 - Sept. 2, (IEEE Computer Society, 2011), pp. 327-331.

[23] D. Dubois and H. Prade, "Abe Mamdani: A pioneer of soft artificial intelligence", in Combining Experimentation and Theory - A Hommage to Abe Mamdani, eds. E. Trillas, P. P. Bonissone, L. Magdalena, and J. Kacprzyk, (Springer, Studies in Fuzziness and Soft Computing, vol. 271, 2012) pp. 49-60.

[24] D. Dubois and H. Prade, "Possibilistic logic. An overview", in Handbook of The History of Logic. Vol. 9 Computational logic, eds. D. M. Gabbay, J. H. Siekmann, J. Woods, (North-Holland, 2014) pp. 283-342.

[25] D. Dubois and H. Prade, "Being consistent about inconsistency: Toward the rational fusing of inconsistent propositional logic bases", in The Road to Universal Logic. Festschrift for the 50th Birthday of Jean-Yves Béziau. Vol.II, eds. A. Koslow, A. Buchsbaum, (Birkhäuser, 2015) pp. 565-571.

[26] D. Dubois and H. Prade, "A possibilistic analysis of inconsistency", Proc. 9th Int. Conf. on Scalable Uncertainty Management (SUM'15) (Ch. Beierle, A. Dekhtyar, eds.), Québec City, Sept. 16-18, (Springer, LNCS 9310, 2015) pp. 347353.

[27] D. Dubois, H. Prade and S. Schockaert, "Stable models in generalized possibilistic logic", Proc. 13th Int. Conf Principles of Knowledge Representation and Reasoning (KR'12) eds. G. Brewka, Th. Eiter, S. A. McIlraith, Rome, June 10-14, (AAAI Press, 2012) pp. 519-529. 
[28] D. Dubois, H. Prade, L. Ughetto, "Fuzzy logic, control engineering and artificial intelligence', in Fuzzy Algorithms for Control, eds. H.B. Verbruggen, H.-J. Zimmermann and R. Babuska, (Kluwer Acad., 1999) pp. 17-57.

[29] A. Hunter, "Reasoning with conflicting information using quasi-classical logic", J. of Logic and Computation 10 (2000) 677-703.

[30] S. Link and H. Prade, "Relational database schema design for uncertain data", Res. Rep. 469, Centre for Discrete Math. and Theoretical Comp. Sci., Univ. of Auckland, August 2014.

[31] E. H. Mamdani and S. Assilian, "An experiment in linguistic synthesis with a fuzzy logic controller", Int. J. of Man-Machine Studies 7 (1975) 1-13.

[32] O. Pivert and H. Prade, "Detecting Suspect Answers in the Presence of Inconsistent Information", Proc. Yth Int. Symp. on Foundations of Information and Knowledge Systems (FoIKS'12), eds. Th. Lukasiewicz and A. Sali, Kiel, March 5-9, (Springer, LNCS 7153, 2012) pp. 278-297.

[33] H. Prade, "A computational approach to approximate and plausible reasoning with applications to expert systems", IEEE Trans. Pattern Anal. Mach. Intell. $7(3)$ (1985) 260-283; "Corrections", IEEE Trans. Pattern Anal. Mach. Intell. 7 (6) (1985) 747-748.

[34] H. Prade and M. Serrurier, "Bipolar version space learning", Int. J. Intell. Syst. 23(10) (2008) pp. 1135-1152.

[35] L. A. Zadeh, "Fuzzy sets", Information and Control 8 (1965) 338-353.

[36] L. A. Zadeh, "Similarity relations and fuzzy orderings", Information Sciences $\mathbf{3}$ (1971) 177-200.

[37] L. A. Zadeh, "Fuzzy sets as a basis for a theory of possibility", Fuzzy Sets and Systems 1 (1978) 3-28.

[38] L. A. Zadeh, "PRUF - A meaning representation language for natural languages", Int. J. Man-Machine Studies 10 (1978) 395-460.

[39] L. A. Zadeh, "A theory of approximate reasoning", in Machine Intelligence, vol. 9, eds. J. E. Hayes, D. Mitchie, and L. I. Mikulich (Ellis Horwood, 1979) pp. 149-194. 